apuntesuniversitarios.upeu.edu.pe

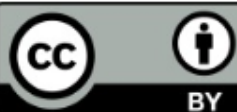

Apuntes Universitarios, 2020: 10(4), octubre-diciembre

ISSN: 2304-0335 DOI:https://doi.org/10.17162/au.v10i4.492

\title{
Enfoque de género y relaciones sociales en el aprendizaje social de estudiantes de nivel secundario
}

\section{Gender and social relations approach in the social learning of secondary school students}

\author{
Manuel Gilberto Ricaldi Ricaldi1a ${ }^{1 a}$, Sebastián Sánchez Díaz ${ }^{2}$ y \\ María Eleonora Subiría Rodríguez ${ }^{3}$ \\ Universidad Cesar Vallejo, Perú ${ }^{123}$ \\ Orcid ID: https://orcid.org/0000-0003-2340-3294 ${ }^{1}$ \\ Orcid ID: https://orcid.org/0000-0002-0099-7694² \\ Orcid ID: https://orcid.org/0000-0002-8053-7646³
}

Recibido: 12 de enero de 2020

Aceptado: 17 de junio de 2020

\begin{abstract}
Resumen
La presente investigación buscó determinar la influencia del enfoque de género y las relaciones sociales en el aprendizaje social de estudiantes de quinto año del colegio Francisco Bolognesi. La hipótesis planteo que existía una influencia significativa del enfoque de género y las relaciones sociales en el aprendizaje social de los estudiantes. La metodología se fundamentó en un enfoque cuantitativo con un diseño no experimental de nivel correlacional-causal. La muestra se compuso de 142 estudiantes. Se utilizó la técnica de la encuesta y los instrumentos fueron 3 cuestionarios. El resultado de la prueba de hipótesis determino que el aprendizaje social depende el $20.8 \%$ del uso del enfoque de género y las relaciones sociales en estudiantes, el valor del Chi cuadrado es de 23.6 y p. valor es igual a 0,000 frente a la significación estadística $\alpha$ igual a 0.05 , significo rechazo de la hipótesis nula. Se concluyó que el enfoque de género y las relaciones sociales en el aprendizaje social están en una tendencia de logro, representando una influencia significativa en el aprendizaje social de los estudiantes de quinto año del colegio Francisco Bolognesi. Los resultados servirán para mejorar el aprendizaje desde una visión inclusiva y social en el ámbito educativo.
\end{abstract}

Key Words: gender studies, social relations, inclusion, learning, education

${ }^{\mathrm{a} C o r r e s p o n d e n c i a ~ a l ~ a u t o r ~}$

E-mail: manuelricaldi@hotmail.com 


\begin{abstract}
This research sought to determine the influence of the gender approach and social relations on the social learning of fifth-year students at the Francisco Bolognesi School. The hypothesis stated that there was a significant influence of the gender approach and social relations on students' social learning. The methodology was based on a quantitative approach with a nonexperimental design of correlational-causal level. The sample was 142 students. The survey technique was used and the instruments were 3 questionnaires. The result of the hypothesis test determined that social learning depends $20.8 \%$ on the use of the gender approach and social relations in students, the Chi-square value is 23.6 and p. value is equal to 0.000 against the statistical significance $\alpha$ equal to 0.05 , meaning rejection of the null hypothesis. It was concluded that the gender focus and social relations in social learning are in a trend of achievement, representing a significant influence in the social learning of fifth year students of the Francisco Bolognesi School. The results will serve to improve learning from an inclusive and social vision in the educational field.
\end{abstract}

Keywords: focus, gender, relationships, social, learning

\title{
Introducción
}

Esta investigación utilizó los postulados teóricos de "genero" desde una visión epistemológica de corte social y reivindicador a partir de los estudios de Sommer (1994), quien afirmaba que era una falacia negar las diferencias biológicas entre los sexos y se alejaba de los postulados teóricos de la llamada "ideología de género" la cual es usada por el actual feminismo radical que solo busca victimizar a la mujer a través del llamado "patriarcado" y busca homogenizar toda diferencia natural y biológica entre los sexos sin un basamento científico que pruebe esta teoría.

A pesar de esta disyuntiva actual, la lucha formal de las mujeres por lograr ser reconocidas socialmente se inició a mediados del siglo XVIII a través de los movimientos sociales conocidos como “olas del feminismo”. Según Bell, Meriläinen y Tienari (2018) los informes en cuanto al desarrollo histórico de los feminismos a menudo utilizan la metáfora de las "olas", lo que hace deducir que las acciones feministas son previas a la presencia de un movimiento social racional y unificador. En la primera y la segunda ola, la lucha feminista se centró en permitir el sufragio, el ingreso activo a la vida política y el derecho de la mujer a una educación sin exclusión social que generara el aumento gradual de la escolarización y el ingreso a la educación superior. Es decir, reconocer a la mujer en un rol activo dentro de la política, la educación y la cultura en la sociedad de esa época. Sommers (1994) refirió que las feministas de la primera ola lucharon por un trato equitativo e igualdad de oportunidades en lo político y educativo, además que rechazaron toda idea de superioridad masculina; por lo que no se sintieron atraídas a crear un 
conflicto entre sexos, ni manifestaron ideas erróneas de superioridad femenina frente a los hombres.

Esta lucha reivindicativa de las mujeres por una educación de equidad a nivel mundial, toma características propias según la idiosincrasia social de cada país al contextualizarla a nivel regional, y aterriza finalmente en el centro principal del aprendizaje que es el aula; donde se genera relaciones sociales de género entre hombres y mujeres. Ante esto, la teoría del aprendizaje social de Bandura (1971) reconoció que todo aprendizaje se basa en constructos sociales, es decir, el aprendizaje se construye por la interacción de los individuos en un entorno real. Wascher et al., (2018) agregaron que la unión entre las relaciones sociales y las diferencias individuales dentro del aprendizaje se han estudiado ampliamente en investigaciones correlaciónales de carácter social. Esto significa que existe una relación fundamental entre género y relaciones sociales.

En el Perú, el sistema educativo no es ajeno a esta problemática social; es así que en Limaen el colegio Francisco Bolognesi precisamente-al analizar la problemática institucional anual, se concluyó que los maestros utilizaban pocas estrategias de enseñanza/aprendizaje adecuadas para desarrollar sesiones pedagógicas que potenciaran el nuevo enfoque transversal de género, sus actitudes y comportamientos observables. Además, los estudiantes presentaban un rendimiento académico medio-bajo al trabajar en grupos mixtos y había una mediana capacidad de relacionarse socialmente; es decir, no eran constantes la relaciones entre grupos heterogéneos, sin discriminación y respetando a todos por igual y se notaba un déficit de coordinación adecuada entre profesores para establecer programas curriculares homogéneos que permitan optimizar un aprendizaje social y humanista establecido dentro del nuevo currículo nacional y sus enfoques para la convivencia y género. En conclusión, la problemática del género y las buenas relaciones sociales eran un problema institucional. Mill (1860) manifestó que las buenas relaciones sociales entre los sexos son fundamentales para lograr el progreso de la sociedad.

Por otro lado, existen algunos antecedentes internacionales referidos al problema de género y relaciones sociales en el ámbito educativo. Villegas (2018), quien realizo una investigación para determinar la relación entre los conceptos de género y la convivencia escolar en estudiantes de una unidad educativa. La metodología se basó en principios cualitativos desde una perspectiva socio crítica de profundidad correlacional. La muestra fue de 120 estudiantes y el instrumento fue una escala tipo Likert. Como principal resultado se establece que existe una 
relación altamente positiva entre las variables. También, Hernández (2017) realizó una tesis cuyo objetivo fue extender el conocimiento existente sobre las relaciones ente iguales desde un enfoque intragénero e intergénero. La metodología fue descriptiva-correlacional de corte transversal. La muestra la conformaron 755 estudiantes de 8 a 13 años a los que se les aplico dos pruebas socio métricas y cuestionarios estructurados. En los resultados se observó notoria influencia del género para diferenciar las relaciones interpersonales entre chicos y chicas y se confirmó la presencia de estereotipos de género en las relaciones entre iguales. Finalmente, Pinto (2016) realizo una tesis cuyo objetivo fue investigar las relaciones de género entre estudiantes en el contexto educativo. La metodología fue cuantitativa con un diseño cuasiexperimental de corte descriptivo, la muestra fue de 722 estudiantes, el instrumento fue un test socio métrico, el resultado de los análisis demuestran que el alumnado adolescente muestra patrones preferenciales de género en el establecimiento de relaciones en el contexto escolar según el tipo de actividad en la que interactúan, estando estos patrones preferenciales altamente condicionados por el género de las personas.

En Perú, Solari (2017) elaboró un estudio cuyo objetivo fue discutir sobre ideología de género, la diversidad en el aula y explorar nuevas relaciones sociales a partir de la interacción docente-estudiantes. La metodología del estudio fue una revisión documental del currículo de educación del 2016. La principal conclusión a partir del análisis de la documentación fue que el enfoque de género incorporado de forma transversal en el currículo nacional constituye un gran aporte en el discurso de equidad para combatir la discriminación, exclusión y violencia que padecen las mujeres. Además, Briones (2017) realizo una investigación cuyo objetivo fue determinar la diferencia de habilidades sociales según el género en estudiantes del nivel secundario. La metodología del estudio fue cuantitativa no experimental, de corte transversal, de tipo descriptivo comparativo. La muestra estuvo conformada por 123 varones y 114 mujeres y el instrumento fue el test de EHS escala de habilidades sociales. La principal conclusión demuestra que existe diferencia significativa de las habilidades sociales según el género en estudiantes del nivel secundario. Por último, Cárdenas (2016) desarrolló una investigación cuyo objetivo fue interpretar la naturaleza y proyección del empoderamiento femenino desde el liderazgo educativo en los estudiantes de secundaria en Perú. La metodología del estudio fue cualitativa con un estudio de casos. La muestra estuvo conformada por estudiantes, padres de familia y docentes y el instrumento fue una entrevista. La principal conclusión demuestra que el liderazgo femenino no es relevante en la institución, predominando la figura masculina en la participación y en la toma de decisiones. Por lo expuesto, la investigación busco determinar la 
influencia del enfoque de género y las relaciones sociales en el aprendizaje social de los estudiantes desde una visión integral entre estudiantes.

Partiendo del fundamento teórico del estudio y presentados los antecedentes sobre género y relaciones sociales; la investigación busco alejarse de los postulados teóricos del feminismo radical que usa la dialéctica marxista de la lucha de clases pero replanteada en una lucha de sexos. Federici (2018) expresó que en la década de los 70s fueron redescubiertos los trabajos de Marx por las feministas que buscaban una teoría prioritaria que pudiera explicar las raíces de la opresión masculina y su lucha contra las tareas domésticas y la dependencia masculina.

Es por esta razón que la investigación toma los constructos teóricos de Christina Hoff Sommers académica y filósofa del "feminismo disidente" surgido en los Estados Unidos, cuyo objetivo principal es volver a las raíces de la lucha de las mujeres, es decir equiparar derechos y oportunidades entre hombres y mujeres y empoderar a las mujeres respetando las diferencias naturales y biológicas que cada uno posee. Sommers (1994) señalo que tomo sus postulados de las feministas de la primera ola, quienes iniciaron la lucha por la paridad, el empoderamiento, la equidad y la igualdad de oportunidades sin restricciones, tabúes o discriminación para ambos sexos en la vida diaria. Punt (2018) afirmo que los movimientos de finales del siglo XX sobre la teoría feminista, los estudios de género a través de la historia social y los proyectos de "recuperación de la mujer" mostraron la necesidad y la conveniencia de un enfoque diferente de género.

Al hablar de género y su asociación con las relaciones sociales, la teoría cognitiva social y la teoría del aprendizaje social de Bandura son fundamentales para entender estas relaciones que surgen de la interacción social, ambas teorías enfatizaban la observación como una forma de aprender. Bussey (2018) señalo que la teoría cognitiva social se desarrolló a partir de la teoría del aprendizaje social. La teoría cognitiva social coincide en muchos preceptos con la teoría del aprendizaje social pero enfatiza en la relación de los procesos socio cognitivos.

Según Bandura (2001) la teoría cognitiva social explicaba la actividad humana a través de una causalidad reciproca tríadica, en la cual intervenían los factores personales (cognitivos, afectivos y bilógicos), patrones conductuales y el medio ambiente, determinado, interactuando e influenciándose el uno al otro bidireccionalmente. Fuentes (2018) amplio la idea ya que señalo que la conducta, los factores personales y los factores medioambientales interactúan entre sí, 
configurando una triangulación dinámica y se basaban en un concepto: la autoeficacia, es decir la creencia en la capacidad de organizar y ejecutar una tarea ante una situación planteada.

Esta interacción del enfoque de género y las relaciones sociales producen un aprendizaje social. Bandura (1971) en su teoría del aprendizaje social indico que el conocimiento se producía por la interacción social a través de procesos de observación e imitación de modelos con la intervención lógica de procesos cognitivos para su procesamiento. Además, agrego que la teoría del aprendizaje social asumía que las influencias del modelado producen aprendizaje principalmente a través de sus funciones informativas y que las observaciones adquieren principalmente representaciones simbólicas de actividades modeladas en lugar de asociaciones específicas de estímulo-respuesta. Horsburgh e Ippolito (2018) señalaron que Bandura, en su teoría del aprendizaje social, proporcionaba un marco útil para considerar cómo aprenden los estudiantes a través del aprendizaje observacional y modelización.

Tomando la teoría cognitiva de Bandura (1989) se puede explicar la forma como se adquiere y se mantiene el género. Adquirir una singularidad en el género es similar a la de cualquier otro comportamiento. Mediante la combinación de observación, imitación y refuerzo diferencial, niños y niñas aprenden a comportarse de acuerdo al grupo social en el que están incluidos. En este proceso de aprendizaje, el comportamiento diferencial de los padres hacia sus hijos juega un papel esencial, de ahí el interés que la perspectiva de género ha puesto en las prácticas de socialización en el aula. Además, Bandura enfatizó el papel del modelo adulto en la transmisión social, es decir, la socialización a través de modelos.

Conceptualmente el termino "género" según la corte penal internacional en el Estatuto de Roma (1998) artículo 7.3 se refiere a los dos sexos masculino y femenino. En el contexto de la sociedad el termino género no tendrá más significado que el anterior. Paveau (2018) definió género como un hecho de la existencia y un enfoque metódico de este contexto. El enfoque de género o perspectiva de género es una visión analítica de la problemática de género. Solís (2016) señalo que el enfoque de género se basaba en la teoría de género relacionada al paradigma histórico-crítico cuyo objetivo era analizar y comprender las relaciones sociales de género para generar una transformación social profunda.

Las relaciones sociales aluden al concepto de interacción entre hombres y mujeres. Bandura (1971), definió las relaciones sociales como relaciones interactivas entre las características personales y las influencias ambientales, las competencias cognitivas se desarrollan y 
modifican por las influencias sociales que transmiten información y activan reacciones emocionales a través del modelado, la instrucción y persuasión social.

El aprendizaje social según Bandura (1971) era un proceso cognitivo que tiene lugar únicamente en un contexto social a través de la observación o la instrucción directa, incluso en ausencia de reproducción motora o refuerzo directo. Juwel y Ahsan (2019) indicaron que el aprendizaje social es un mecanismo individual o acción colectiva para cambiar en comprensión, comportamiento o agenda a través de la interacción entre actores de una red particular.

Epistemológicamente, la investigación utilizo el conocimiento producido sobre enfoque género y relaciones sociales en el contexto social para explicar científicamente los factores que intervinieron en ella, como se manifestaron estos fenómenos, analizarlos y producir conocimiento científico valido para su divulgación académica. Se apoyó en el paradigma positivista, el cual está fuertemente ligado a la realidad social.

En la práctica, la investigación determinó que el enfoque de género y las relaciones sociales son importantes para la mejora del aprendizaje educativo desde una visión social, siendo de utilidad práctica inmediata en programas educativos con fuerte carga humanista y social direccionada a reconfigurar un nuevo horizonte social de relaciones positivas e igualitarias de género. Metodológicamente, el interés de la presente investigación fue explicar el tema de género en la sociedad, por lo que utilizo un diseño no experimental con enfoque positivista. Teóricamente, la investigación se fundamentó en teorías con fuerte carga filosófica-humanista para comprender desde una visión global los fenómenos sociales de género y el discurso dialectico valido de legitimación social de las mujeres. Por lo expuesto anteriormente el objetivo de la investigación fue determinar la influencia del enfoque de género y las relaciones sociales en el aprendizaje social de estudiantes de quinto año del colegio Francisco Bolognesi, Lima 2019.

\section{Metodología}

La investigación utilizo el enfoque cuantitativo y el paradigma positivista. Rubtcova, Martianova, y Kaisarova (2019) indicaron que el positivismo pertenecía al realismo sociológico, ya que reconocía la existencia real de la sociedad y su supremacía sobre el individuo. El método de esta investigación fue hipotético-deductivo. Fue de tipo básico, porque busco ampliar el conocimiento real y comprender mejor el suceso a través de un diseño no experimental de corte transversal sin manipulación de las variables ni experimentación por lo 
que los datos fueron recolectados en un solo momento. El nivel de la investigación correlacional-causal estuvo orientado a analizar las variables independientes (causales) y sus resultados verificables en la variable dependiente (efecto), es decir a un cambio en las variables independientes correspondió un cambio en la variable dependiente.

La población total estuvo conformada por 225 estudiantes de $5^{\circ}$ año de secundaria de las secciones A-B-C-D-E-F-G del colegio Francisco Bolognesi ubicado en la provincia de Lima, departamento de Lima. Al ser una investigación social se consideró algunos criterios antes de seleccionar la muestra: la edad de maduración social de los estudiantes, la exposición del enfoque transversal de género en las sesiones de aprendizaje, el nivel de apreciación critica del tema y fundamentalmente el consentimiento informado para participar voluntariamente en la investigación. Luego de considerar estos parámetros para obtener una muestra representativa se utilizó el muestreo probabilístico, de tipo aleatorio estratificado con sub divisiones identificadas en las secciones de la población de estudiantes utilizando una fórmula estadística para poblaciones finitas con un nivel de confianza al 95\% y un error máximo al 5\%, aplicando la formula se obtuvo un resultado de 142 estudiantes para la muestra.

La técnica fue la encuesta y los instrumentos aplicados, durante el segundo semestre del año 2019 a los 142 estudiantes que conformaron la muestra, fueron tres cuestionarios estructurados. El primer cuestionario para medir la variable enfoque de género se denominó "Cuestionario sobre enfoque de género" El segundo, para medir la variable relaciones sociales, se denominó "Cuestionario sobre relaciones sociales" y el tercero, para medir la variable aprendizaje social se denominó "Cuestionario sobre aprendizaje social”, todos de autoría propia y con 90 ítems cada uno. La validez de contenido la determino la evaluación de 5 jueces que concluyeron que los instrumentos poseían pertinencia, relevancia y claridad por lo tanto eran aplicables. La evaluación métrica de los instrumentos se inició con la prueba piloto a una muestra 50 estudiantes que determino un porcentaje favorable de claridad en la redacción de los ítems existiendo una correlación favorable de los puntajes obtenidos mediante el procedimiento correlación -ítem- total. Finalmente el estadístico del Alfa de Crombach determinó una confiabilidad final de $92,1 \%, 90,0 \%$ y $90,1 \%$ respectivamente para cada instrumento por lo tanto se concluyó que fueron altamente confiables. La prueba de normalidad de KolgomorovSmirnov a los datos obtenidos determino que no existe distribución normal de los datos al obtener menos a 0,00 de significación $<0,05$ p valor por lo que para el análisis estadístico se utilizó una prueba no paramétrica de regresión ordinal. 
La investigación empleo tres variables por lo que estadísticamente necesito un análisis multivariante o multivariado direccionado al análisis deductivo, confirmatorio de estructura causal utilizando el paquete estadístico SPSS 25. Roldán y Fachelli (2015) expresaron que el análisis multivariable utilizabas métodos y técnicas para el análisis estadístico simultaneo de relaciones entre tres a mas variables. Los resultados estadísticos se presentaron descriptiva e inferencialmente. Martín (2012) expreso que la estadística descriptiva describía la información analizada utilizando el cálculo, la dispersión o las tendencias para obtener conclusiones de esto datos y la estadística inferencial para realizar inferencias estadísticas y la prueba de hipótesis.

\section{Resultados}

Según la tabla 1 y figura 1, se observó que cuando el aprendizaje social está en un nivel de inicio al $0.7 \%$, el enfoque de género está un nivel de logro al $0.7 \%$, cuando el aprendizaje social está en proceso al $7.7 \%$ el enfoque de género está en nivel de logro al $21.1 \%$ y cuando el aprendizaje social está un nivel de logro al 2,8\% el enfoque de género está en nivel de logro al $66.9 \%$. De los resultados en conjunto podemos concluir que cuando el enfoque de género está en nivel de logro el aprendizaje social está en un nivel de proceso en los estudiantes.

\section{Tabla 1}

Tabla cruzada de aprendizaje social y enfoque de género

\begin{tabular}{|c|c|c|c|c|c|}
\hline & & & Enfoque & Genero & Total \\
\hline & & & Proceso & Logro & \\
\hline \multirow[t]{6}{*}{ Aprendizaje Social } & Inicio & Recuento & 1 & 1 & 2 \\
\hline & & $\%$ del total & $0,7 \%$ & $0,7 \%$ & $1,4 \%$ \\
\hline & Proceso & Recuento & 11 & 30 & 41 \\
\hline & & $\%$ del total & $7,7 \%$ & $21,1 \%$ & $28,9 \%$ \\
\hline & Logro & Recuento & 4 & 95 & 99 \\
\hline & & $\%$ del total & $2,8 \%$ & $66,9 \%$ & $69,7 \%$ \\
\hline \multirow[t]{2}{*}{ Total } & & Recuento & 16 & 126 & 142 \\
\hline & & $\%$ del total & $11,3 \%$ & $88,7 \%$ & $100,0 \%$ \\
\hline
\end{tabular}




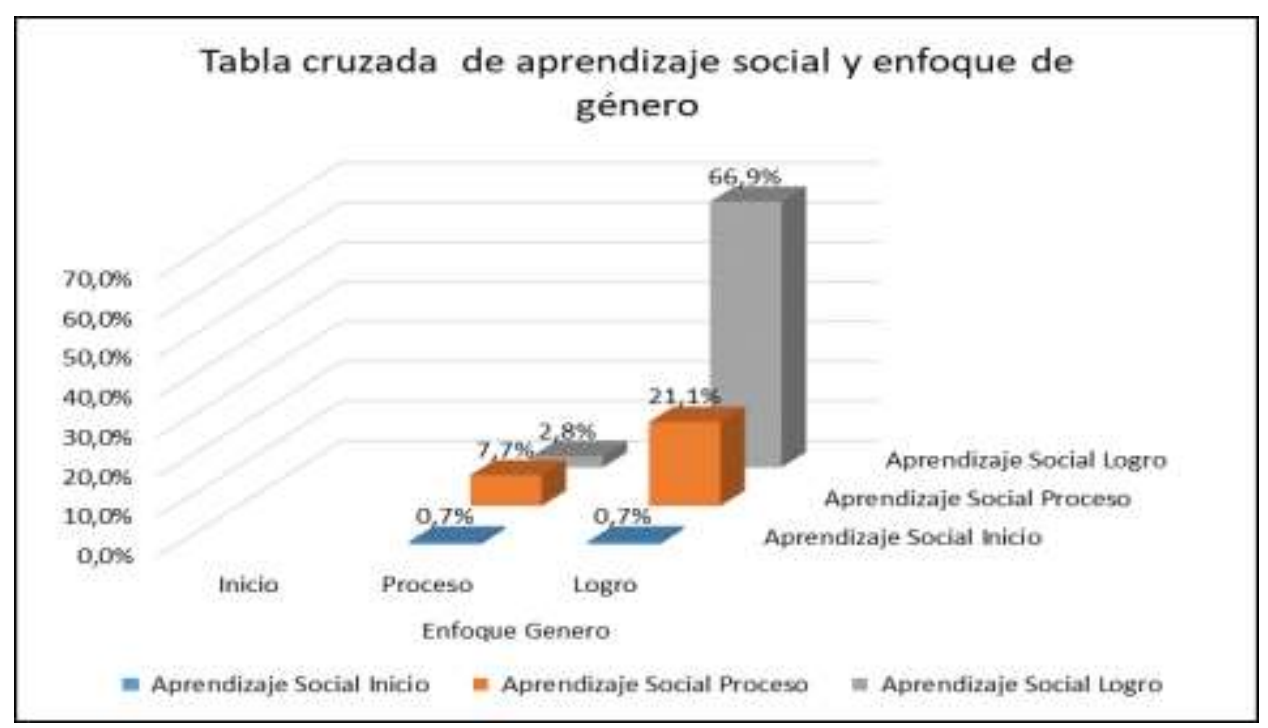

Figura 1: Grafica de tabla cruzada de aprendizaje social y enfoque de género.

Según la tabla 2 y figura 2, se observó que cuando el aprendizaje social está en un nivel de inicio al $0.0 \%$, las relaciones sociales están en nivel regular al 1.4\%, cuando el aprendizaje social está en proceso al $7.0 \%$, las relaciones sociales están en un nivel bueno al 21,8 .0\% y cuando el aprendizaje social está un nivel de logro al 1,4, \%, las relaciones sociales están en nivel bueno al $63.8 \%$.

Tabla 2

Tabla cruzada de aprendizaje social y relaciones sociales

Tabla cruzada Aprendizaje Social ${ }^{\star}$ Relaciones Sociales

\begin{tabular}{lllrrr}
\hline & & \multicolumn{2}{c}{ Relaciones Sociales } & Total \\
\hline Aprendizaje Social & Inicio & Recuento & 0 & Bueno & 2 \\
& & \% del total & $0,0 \%$ & $1,4 \%$ & $1,4 \%$ \\
& Proceso & Recuento & 10 & 31 & 41 \\
& & $\%$ del total & $7,0 \%$ & $21,8 \%$ & $28,9 \%$ \\
& \multirow{2}{*}{ Logro } & Recuento & 2 & 97 & 99 \\
& & \% del total & $1,4 \%$ & $68,3 \%$ & $69,7 \%$ \\
Total & & Recuento & 12 & 130 & 142 \\
& & $\%$ del total & $8,5 \%$ & $91,5 \%$ & $100,0 \%$ \\
\hline
\end{tabular}




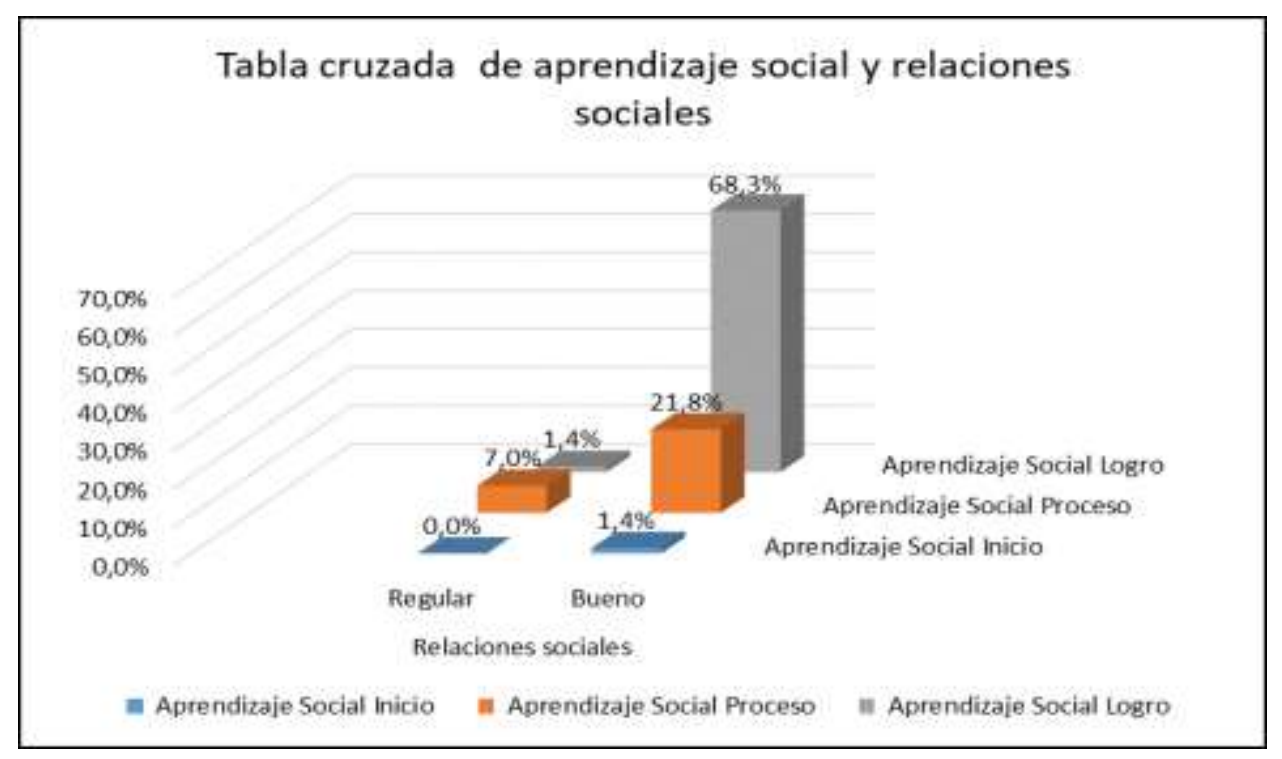

Figura 2: Grafica de tabla cruzada de aprendizaje social y relaciones sociales.

De los resultados en conjunto podemos concluir que cuando las relaciones sociales están en nivel de bueno el aprendizaje social está en un nivel de proceso en los estudiantes de quinto año del colegio Francisco Bolognesi.

\section{Prueba de hipótesis}

$\mathrm{H}_{\mathrm{o}}$ : El enfoque de género y las relaciones sociales no influyen significativamente en el aprendizaje social de estudiantes de quinto año del colegio Francisco Bolognesi, Lima 2019.

$\mathrm{H}_{1}$ : El enfoque de género y las relaciones sociales influyen significativamente en el aprendizaje social de estudiantes de quinto año del colegio Francisco Bolognesi, Lima 2019.

Según la tabla 3 en cuanto de la prueba del pseudo R cuadrado, lo que se estarían presentando es la dependencia porcentual del enfoque de género y las relaciones sociales en el aprendizaje social en los estudiantes de quinto año del colegio Francisco Bolognesi el cual se tiene al coeficiente de Nagalkerke, implicando que la variabilidad del aprendizaje social depende el $20.8 \%$ del uso del enfoque de género y las relaciones sociales en estudiantes. 


\section{Tabla 3}

Pseudo coeficiente de determinación de las variables.

\begin{tabular}{ll} 
Cox y Snell &, 154 \\
Nagelkerke &, 208 \\
McFadden &, 124 \\
\hline
\end{tabular}

Según la Figura 3 de la representación del área COR como incidencia del enfoque de género y las relaciones sociales en el aprendizaje social. En cuanto al resultado de la curva COR, se tiene el área que representa la capacidad de clasificación de un 75.7\% representando un nivel alto de implicancia del enfoque de género y las relaciones sociales que influyen en el aprendizaje social de estudiantes de quinto año del colegio Francisco Bolognesi, Lima 2019.

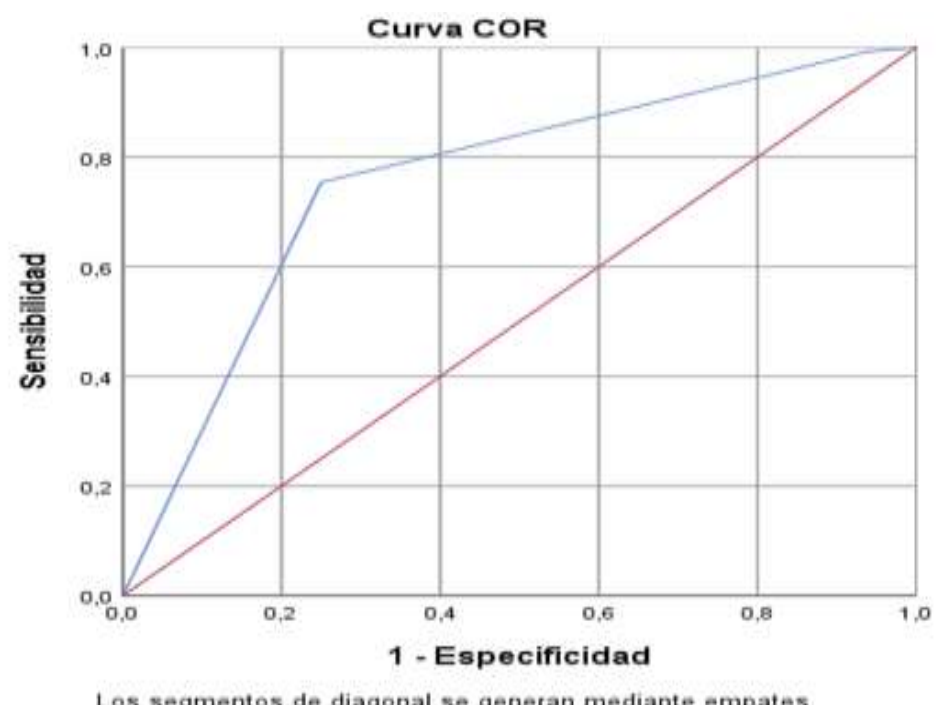

\section{Área bajo la curva: 0,757}

Figura 3: Representación del área COR como incidencia del enfoque de género y las relaciones sociales en el aprendizaje social.

\section{Discusión}

Según los resultados de la investigación de Villegas (2018) se estableció que existe una relación altamente negativa de convivencia escolar; el 54\% de estudiantes cree que es nada favorable, el $31 \%$ cree que es poco favorable y el $15 \%$ cree que es favorable, lo que significa que existen dificultades en las relaciones interpersonales entre los estudiantes. Sobre la concepción de género el $52 \%$ cree que es nada equitativa, el $33 \%$ cree que es poco equitativa y 
el $15 \%$ cree que es equitativa, lo que significa que existen dificultades en el concepto de género de igualdad y equidad entre los y las adolescentes sobre el trato igualitario.

Desde los resultados descriptivos de la presente investigación se observó que los estudiantes manifestaron en un 88,73\% que el enfoque de género está en nivel de logro, el 91,55\% que las relaciones sociales están en un nivel bueno y el 69,72 \% que el aprendizaje social está en el nivel de logro. Se concluyó que el enfoque de género y las relaciones sociales en el aprendizaje social están en una tendencia de logro en los estudiantes del colegio Francisco Bolognesi. Estos resultados demuestran que un enfoque de género alejado de postulados teóricos de confrontación es relevante para una convivencia social aceptable a nivel del aula. Paglia (2018) afirmo que el patriarcado no existe y exigió a las mujeres a no vivir como víctimas. Sommers (1994) realizo una crítica fuerte del feminismo ideológico actual el cual busca confrontar y no unir a todos por igual.

El objetivo principal de Villegas (2018) fue determinar la relación entre los conceptos de género y la convivencia escolar: Partió desde la teoría socio-crítica para demostrar como los fenómenos de la sociedad afectan a los y las estudiantes, tanto física como psicológicamente, convirtiendo el colegio un ambiente hostil y que se ve reflejado una conducta insegura a nivel social. No se concuerda con esta visión teórica de la realidad social. Bandura (1971) señaló que, en gran medida, en la conducta influyen los factores sociales y el contexto social en el desarrollo de la personalidad ya que el medio ambiente aporta modelos y que, si la conducta del modelo corresponde a la situación real de los individuos (intereses, experiencias, metas), el individuo imitará la conducta del modelo.

Según los resultados de la investigación de Hernández (2017) se observó notoria influencia del género para diferenciar las relaciones interpersonales entre chicos y chicas y se confirmó la presencia de estereotipos de género en las relaciones entre iguales. El análisis correlacional entre los diferentes índices sociométricos intra e intergénero determino una correlación media alta entre las variables. La medida de adecuación muestral KMO (Kaiser-Meyer-Olkin) y la prueba de esfericidad de Barlett aportan significación a la extracción de los factores $(\mathrm{KMO}=.666$, prueba de esfericidad de Barlett=6290,466(190), $\mathrm{p}<.001)$.

Los resultados de la investigación concuerdan con los resultados de Hernández (2017) sobre la influencia del género en las relaciones sociales según los resultados de la prueba de hipótesis, pseudo R cuadrado se obtuvo al coeficiente de Nagalkerke, implicando que la variabilidad del 
aprendizaje social depende el $20.8 \%$ del uso del enfoque de género y las relaciones sociales en estudiantes, representando un nivel alto de implicancia del enfoque de género y las relaciones sociales que influyen en el aprendizaje social de estudiantes de quinto año del colegio Francisco Bolognesi.

Hernández (2017) se respaldó en la teoría de Rebollo et al. (2011). Estos autores expresaron que, a fin de comprender y analizar el género, se deben considerar tres perspectivas: primero, la que entiende el género como un sistema de organización social; segunda, la del género como un proceso dinámico de representación de lo que significa ser hombre o mujer; tercero, la del género como un aspecto de la identidad y de las actitudes personales. A partir de la teoría de Sommers (1994), se señalan cuatro aspectos fundamentales sobre el género: la paridad, el empoderamiento, la equidad y la igualdad de oportunidades para ambos sexos en la vida diaria. Además, Bandura (2001) explica la actividad humana a través de una causalidad reciproca tríadica, en la cual intervienen los factores personales, patrones conductuales y el medio ambiente, determinando, interactuando e influenciándose el uno al otro bidireccionalmente. Por lo expuesto teóricamente, se concuerda con el análisis sobre el género realizado por Hernández (2017).

Según los resultados de la investigación de Pinto (2016), se concluyó que el alumnado adolecente mostro altos patrones preferenciales de género en el establecimiento de relaciones en el contexto escolar. Los resultados del cálculo del coeficiente Alpha de Cronbach para el conjunto de la EPRG (con el procedimiento reliability del SPSS), demostró un óptimo resultado en consistencia interna (Alpha $=.92)$; lo que significa que los roles de género son muy consistentes y altos en niveles de concordancia grupal entre sí. La prueba de Chi-Cuadrado indico significación en la definición y explicación de su asociación con cada factor calculado. Al ser la distribución de los datos no son paramétricas, se aplicó el estadístico U de MannWhitney lo que determinó que los datos fueron estadísticamente significativos $(\mathrm{p}=0,000)$.

El objetivo de estudio de Pinto (2016) fue investigar las relaciones de género entre estudiantes en el contexto educativo. Desde el objetivo general de la investigación se concuerda significativamente con los resultados de Pinto (2016), ya que se determinó la dependencia del enfoque de género y las relaciones sociales en el aprendizaje social en los estudiantes de quinto año del colegio Francisco Bolognesi, el valor del Chi cuadrado fue de 23.6 y p. valor (valor de significación) es igual a 0,000 frente a la significación estadística $\alpha$ igual a 0.005 ( $\mathrm{p}$. valor $<\alpha$ ). 
Pinto (2016) se respaldó en la teoría de Bosch, Ferrer y Alzamora (2006) quienes afirmaron que el proceso de socialización estaba asignado por roles diferenciados de hombres y mujeres, de tal modo que cada uno se educa de distinta manera. Se discrepó con este postulado teórico. Sommers (1994) afirmaba que las mujeres y los hombres especialmente en el campo de la educación deberían tener un trato equitativo sin ningún tipo de discriminación. Se concordó según sus teóricos que el aprendizaje de la masculinidad y la feminidad entre sí se hacen por influencia social. Para Bandura (1971) en la conducta influyen mucho los factores sociales y el contexto social en el desarrollo de la personalidad.

Según los resultados de la investigación de Solari (2017) la principal conclusión a partir del análisis inferencial de la documentación teórica fue que el enfoque de género incorporado de forma transversal en el currículo nacional constituye un gran aporte en el discurso de equidad para combatir la discriminación, exclusión y violencia que padecen las mujeres. Los resultados de la investigación en el colegio Francisco Bolognesi determino que el empoderamiento está al 90,85\% en el nivel de logro. Y esto evidencia una percepción positiva del empoderamiento en el colegio. Finalmente se observó que el $87,32 \%$ \% de los estudiantes encuestados, manifestó que la equidad está en un nivel de bueno evidenciando que los estudiantes manifestaron una percepción positiva de la equidad ante el género. Por lo que se concuerda con los resultados de Solari (2017).

El principal objetivo de Solari (2017) fue discutir sobre la ideología de género, la diversidad en el aula y explorar nuevas relaciones sociales a partir de la interacción docente-estudiantes; se basó en la teoría de Bertrand-Ricovert (2006) quien indico que la mujer contribuye al reforzamiento de los lazos existentes y a la creación de nuevas relaciones, que hacen del hogar el ámbito privilegiado del ejercicio de la sociabilidad femenina. Se concordó con esta teoría. Bandura (1963) explico que niños y niñas aprenden a comportarse de acuerdo al grupo social en el que están incluidos donde los padres juegan un rol decisivo de ahí el interés que la perspectiva de género ha puesto en las prácticas de socialización en el aula.

Según los resultados de la investigación de Briones (2017) determinó en un nivel de significancia del 5\% que existía diferencia significativa de las habilidades sociales según el género en estudiantes del nivel secundario del Colegio Adventista Puno, 2017. Por lo tanto se rechaza la Ho a favor de la Ha, aceptando la hipótesis general de investigación. 
Desde la investigación en el colegio Francisco Bolognesi-y según el análisis inferencial de los datos-se determinó que el enfoque de género y las relaciones sociales influyen significativamente en el aprendizaje social de los estudiantes de quinto año, ya que el p- valor (valor de significación) es igual a 0,000 frente a la significación estadística $\alpha$ igual a 0.005 (p. valor $<\alpha$ ), lo que significo rechazo de la hipótesis nula y existen evidencias para aceptar la hipótesis alterna. Por lo tanto desde las relaciones sociales se puede concordar con la influencia de las habilidades sociales en el aprendizaje de los estudiantes.

Briones (2017) se basó en la teoría de Caballo (2002), quien estudió la relación entre las habilidades sociales y el aprendizaje desde la influencia del medio ambiente, lo cual puede intervenir en los patrones conductuales o en el proceso de aprendizaje. Bandura (2001) señalo que el aprendizaje se da en un entorno social mediante la interacción de los individuos y son consecuencia de la interacción de influencias personales, de comportamiento y ambientales. Por lo que se concordó con la base teoría de Briones (2017).

Finalmente, según los resultados de la investigación de Cárdenas (2016) del análisis empírico-analítico de la categorización de los concepciones socioculturales de género, estereotipos y formas de relación de genero demostró que el liderazgo femenino no era relevante, predominando la figura masculina en la participación y en la toma de decisiones en la escuela.

Dentro de las conclusiones de la investigación en el colegio Francisco Bolognesi se observo que el $87,32 \%$ de los estudiantes encuestados, manifestó que los factores personales están en un nivel de bueno. Y esto evidencia que los estudiantes manifestaron una percepción positiva de los factores personales en relación al género; además, se observó que el 90,85\% de los estudiantes encuestados, manifestó que el empoderamiento está en un nivel de logro. Y esto evidencia que los estudiantes manifestaron una percepción positiva del empoderamiento. Por lo observado no se concuerda con los resultados obtenidos por Cárdenas (2016).

El objetivo principal de Cárdenas (2016) fue interpretar la naturaleza y proyección del empoderamiento femenino desde el liderazgo educativo en estudiantes de $5^{\circ}$ año de secundaria en Perú y se basó en la teoría de Lewin (citado por Torrecilla, 2006), quien afirmó la existencia de un liderazgo autoritario desde un enfoque de género dominante y con tendencia al maltrato psicológico y físico a las mujeres, lo que nos les permite empoderase para ser líderes. No se concuerda con esta teoría. Sommers (1994) explico que la mujer debía considerar su papel de 
líder y eliminar conceptos de debilidad, esto lo reafirman Chinwokwu y Arop (2018) que mencionaron que la mujer debe verse ella misma capaz de realizar cualquier tarea y eliminar estereotipos o sexismos.

Se puede inferir — según los resultados obtenidos sobre género-que el factor humano, al ser una investigación social, es un limitante en el desarrollo y las conclusiones de la investigación. La edad de maduración social de los estudiantes en temas de género, la confusión de conceptos teóricos entre enfoque de género e ideología de género de los estudiantes al no tener información científica valida en los colegios, representan dificultades al trabajar con adolescentes. Además, el tema de género ha sido incluido con muchas modificatorias y dudas epistemológicas como contenido transversal en las políticas educativas de los ministerios de educación en los países sudamericanos, careciendo del respectivo análisis social de la realidad educativa continental y previa capacitación docente; esta falta de conocimiento genera conflictos cognitivos no solo en los estudiantes sino también en los maestros al trabajar el tema de genero desde un ámbito cultural y social y no-científico. Por lo tanto, se sugiere ampliar las investigaciones sobre género en temas claves como: igualdad de oportunidades, empoderamiento social y equidad de género pero desde una mirada científica-biológica y social desarraigando ideologías de confrontación social.

\section{Conclusiones}

Según el análisis descriptivo, se observó que los estudiantes manifestaron en un 88,73\% que el enfoque de género está en nivel de logro, el 91,55\%, que las relaciones sociales están en un nivel bueno y el 69,72 \% que el aprendizaje social está en el nivel de logro. Se concluye que el enfoque de género y las relaciones sociales en el aprendizaje social en los estudiantes del colegio Francisco Bolognesi están en una tendencia de logro.

Según el análisis inferencial se determinó que el enfoque de género y las relaciones sociales influyen significativamente en el aprendizaje social de los estudiantes de quinto año del colegio Francisco Bolognesi, ya que el p- valor (valor de significación) es igual a 0,000 frente a la significación estadística $\alpha$ igual a 0.005 (p. valor $<\alpha$ ), lo que significa rechazo de la hipótesis nula y existen evidencias para aceptar la hipótesis alterna de la investigación. 


\section{Referencias}

Bandura, A. (1971). Social Learning Theory. New York: General Learning Press.

Bandura, A. (1989). Social Cognitive Theory. Annals of child development, 6(1), 1-60.

Bandura, A. (2001). La teoría cognitiva social: una perspectiva agencial. Revisión anual de Psicología, 7.

Bell, E., Meriläinen, S., \& Tienari, S. T. (2018). Time's up! Feminist theory and activism meets organization studies. Human Relations, 72(1), 4-22.

Bertrand-Ricoveri P. (2010) Mitología Shipibo. Un viaje en el imaginario de un pueblo amazónico. París: L’Harmattan.

Boch, E., Ferrer, V., \& Alzamora, A. (2006). El laberinto patriarcal: reflexiones teóricoprácticas sobre la violencia contra las mujeres. Barcelona: Anthropos.

Briones, Y. (2017). Habilidades sociales según el género en estudiantes del nivel secundario del Colegio Adventista Puno, 2017. (Tesis de pregrado). Universidad Peruana Unión, Lima, Perú

Bussey, K. (2018). Social cognitive theory across the life span. The SAGE Encyclopedia of Lifespan Human Development, 3.

Caballo, V. (2002). Manual de Evaluación y Entrenamiento de las Habilidades Sociales. España: Editorial siglo XXI

Cárdenas Hurtado, L. (2016). Liderazgo femenino de estudiantes en una institución educativa, Perú. Revista Enfoques Educacionales, 13(1) ,105-129.

Estatuto de Roma de la Corte Penal Internacional, Artículo 7.3 (1988)

Federici, S. (2018). Marx and Feminism. tripleC, Communication, capitalism and critique, 8.

Fuentes, L. E. (2018). La teoría del capital social y la teoría social cognitiva en la cantidad y calidad de conocimiento. RECAI Revista de Estudios en Contaduría, Administración e Informática, 30 .

Hernández, C. M. (2017). Las relaciones entre iguales en el alumnado de primaria y secundaria desde una perspectiva de género (tesis doctoral). Universidad de la Laguna, San Cristobal, España.

Horsburgh, J., Ippolito, K. (2018). A skill to be worked at: using social learning theory to explore the process of learning from role models in clinical settings. BMC Medical Education 18(156). 1-8.

Juwel, R., \& Ahsan, A. (2019). Social Learning and Governance in Farazmand. Global Encyclopedia of Public Administration, Public Policy, and Governance. Springer, Cham, 9.

Martín, Z. H. (2012). Métodos de Análisis de Datos. Rioja: Universidad de la Rioja.

Mill, J. S. (1860). The Subjection of Women. London: Longman.

Plagia, C. (2018). Feminismo pasado y presente. Madrid: Turner.

Paveau, M.-A. (2018). Le genre: une épistémologie contributive pour l'analyse du discours. ENS Éditions, 19.

Pinto, E. R. (2016). Género en el Alumnado de Educación Secundaria: Análisis de las Redes Sociales del aula desde una Perspectiva de Género (tesís doctoral). Universidad de Sevilla, Sevilla, España. 
Punt, J. (2018). Gender studies and biblical interpretation: (How) Does theory matter? The African Journal of Gender and Religion, 24(2), 68-94.

Rebollo, Á., García, R., Piedra, J. y Vega, L. (2011). Diagnóstico de la cultura de género en educación: Actitudes del profesorado hacia la igualdad. Revista de Educación, 355(2), 521546.

Roldán, P. L., \& Fachelli, S. (2015). Metodología de la Investigación Social Cuantitativa. Barcelona: Universitat Autònoma de Barcelona.

Rubtcova, M., Martianova, N., \& Kaisarova, V. (2019). The role of women in a positivist society: the transformation of social practices of interaction in Auguste Comte. Revista Dilemas Contemporáneos: Educación, Política y Valores, 6 (3), 1-16.

Solari, R. M. (2017). El Enfoque de Género en el Currículo Nacional: Una aproximación desde la Interculturalidad. Acta Herediana, 60(41), 13-21.

Solís, A. (2016). La perspectiva de género en educación. Desarrollo profesional docente: reforma educativa, contenidos curriculares y procesos de evaluación, 2(5), 97-107.

Sommers, C. H. (1994). Who stole feminism? New York: Simon \& Schuster.

Torrecilla, F. (2006). Una dirección escolar para el cambio: del liderazgo transformacional al liderazgo distribuido. Revista Iberoamericana sobre Calidad, Eficacia y Cambio en Educación, 4(4), 11-24.

Villegas, V. (2018). Concepciones de Género y Convivencia Escolar en los y las estudiantes de la Unidad Educativa Cmdt. César Endara Peñaherrera. Revista Científica Retos de la Ciencia, 2(2), 139-154.

Wascher, C., Kulahci, I., Langley, E., \& Shaw, R. (2018). How does cognition shape social relationships? Philosofical Transactions of the Royal Society B, 373(1756), 1-9. 